\title{
Variable-poled Tracking Control of a Two-wheeled Mobile Robot Using Differential Flatness
}

\author{
Liming Chen, Yingmin Jia \\ The Seventh Research Division, Beihang University (BUAA), \\ Beijing, 100191, China \\ E-mails: clmtest@126.com,ymjia@buaa.edu.cn
}

\begin{abstract}
This paper investigates the tracking control of a two-wheeled mobile robot in both kinematic and dynamic models. Differential flatness and PD-spectral theory are used for controller design. Based on differential flatness, the original system is transformed via a state prolongation and a state transformation into a normal form to apply feedback linearization. Then using PD-spectral theory, variable poles of tracking error dynamics are assigned to realize the stability of trajectory tracking. Simulation results are presented to demonstrate the effectiveness of the proposed method.
\end{abstract}

Keywords: mobile robot, nonlinear control, trajectory tracking, differential flatness

\section{Introduction}

Wheeled mobile robots have been proven to be one of the most active areas of research since they are much useful in varieties of applications ranging from industrial settings, to military systems, to home robotics, etc. One of the main lines of research is the trajectory tracking problem, which is concerned with driving a mobile robot as close as possible to a desired explicit trajectory. And the tracking control approaches include backstepping, ${ }^{1}$ sliding mode control, ${ }^{2}$ linearization, ${ }^{3}$ neural network-based control, $^{4}$ fuzzy control $^{5}$ and differential flatness-based control ${ }^{6-8}$ which is also used in this paper.

Differential flatness has been introduced by Fliess et al. ${ }^{9}$ It is a very useful tool for nonlinear controller design. Roughly speaking, a system is differentially flat, if there exists variables of the same dimension as inputs, called flat output, such that states and inputs can be algebraically expressed in terms of flat output and its derivatives. Moreover, this mapping is invertible, and the system is equivalent to a linear one. If the desired trajectory of flat output is given, then by performing a feedback linearization and designing a time invariant controller for the linearized system around the desired trajectory, stable tracking error dynamics are achieved. Kinematic model and dynamic model of a two-wheeled mobile robot have been proven to be differentially flat by choosing the center position of the wheel axle of the robot as the flat output. In Refs. 7, 8, controllers are designed in this scheme. However, in these controllers, the parameters of the tracking error dynamics are constant, which means that the convergence speed of tracking error is fixed, and this brings limitation for this method.

In this paper, the use of PD-spectral theory of linear time-varying (LTV) systems is proposed for tracking controller design. The PD-spectral theory has been developed by Zhu, ${ }^{10}$ which can be seen as a natural extension of the conventional eigenvalue-eigenvector theory for linear time-invariant (LTI) systems. After the state transformation based on differential flatness, the 
application of PD-spectral theory becomes much easier. By assigning time-varying "poles", more generalized tracking error dynamics can be obtained. And the poles can be changed at any time as we want.

The rest of this paper is organized as follows: In section 2, the kinematic model and dynamic model of a two-wheeled mobile robot are derived and analyzed with differential flatness. Section 3 presents the design of control law in both models. Simulation results are shown in section 4. Finally, concluding remarks are given in section 5 .

\section{Models of Two-wheeled Mobile Robot with Differential Flatness}

\subsection{Kinematic model}

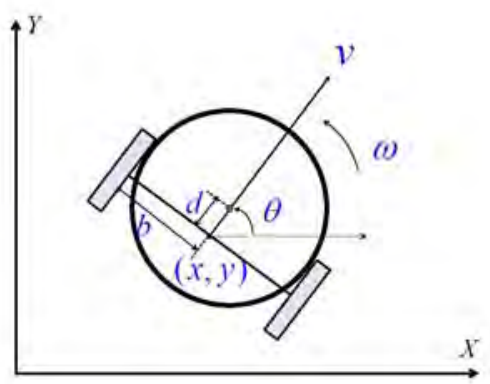

Fig.1. The configuration of a two-wheeled mobile robot with no slip.

Fig. 1 shows that the robot's configuration in Cartesian coordinates is given by $\mathbf{q}=[x, y, \theta]^{T}$ where $(x, y)$ is the coordinates of the center of the wheel axle and $\theta$ is the heading angle of the robot. With the assumption of noslip condition at the wheel contact points, the velocity of the wheel centers are parallel to the heading orientation. Then the kinematic model can be written as

$$
\dot{\mathbf{q}}=S(\mathbf{q}) \mathbf{v}=\left[\begin{array}{cc}
\cos \theta & 0 \\
\sin \theta & 0 \\
0 & 1
\end{array}\right]\left[\begin{array}{c}
v \\
\omega
\end{array}\right]
$$

where, $v$ is the heading speed and $\omega$ turning speed.

To outline how the kinematic model of the twowheeled mobile robot is differentially flat, we need to select suitable flat outputs and express all state variables and inputs in terms of the flat outputs and their derivatives. The dimensions of flat outputs should be equal to that of the inputs. Here, we can choose the center position of the wheel axle $(x, y)$ as the flat outputs $\left(z_{1}, z_{2}\right)$.

With the chosen flat outputs, the states can be expressed as $x=z_{1}, y=z_{2}, \theta=\arctan \frac{\dot{z}_{2}}{\dot{z}_{1}}$, and the two inputs can be written as $v=\sqrt{\dot{z}_{1}^{2}+\dot{z}_{2}^{2}}, \omega=\frac{\dot{z}_{1} \ddot{z}_{2}-\ddot{z}_{1} \dot{z}_{2}}{\dot{z}_{1}^{2}+\dot{z}_{2}^{2}}$. It can be noticed that the expression of state $\theta$ contains the first order derivatives of both the flat outputs, $\dot{z}_{1}$ and $\dot{z}_{2}$. According to differential flatness theory, the system (1) need to be extended to 4 dimensions. Apply one prolongation of $v$ by considering it as an additional state, then the extended system is given by

$$
\begin{array}{ll}
\dot{x}=v \cos \theta, & \dot{v}=u_{1} \\
\dot{y}=v \sin \theta, & \dot{\theta}=u_{2}
\end{array}
$$

where $u_{1}$ and $u_{2}=\omega$ are new inputs of the extended system. The new inputs can be calculated as

$$
u_{1}=\frac{\dot{z}_{1} \ddot{z}_{1}+\dot{z}_{2} \ddot{z}_{2}}{\sqrt{\dot{z}_{1}^{2}+\dot{z}_{2}^{2}}}, u_{2}=\frac{\dot{z}_{1} \ddot{z}_{2}-\ddot{z}_{1} \dot{z}_{2}}{\dot{z}_{1}^{2}+\dot{z}_{2}^{2}} \text {. }
$$

The extended system (2) can be transformed via a state transformation $[x, y, v, \theta]^{T} \leftrightarrow\left[z_{1}, z_{2}, \dot{z}_{1}, \dot{z}_{2}\right]^{T}$ into a normal form

$$
\begin{aligned}
& \ddot{z}_{1}=u_{1} \cos \left(\arctan \frac{\dot{z}_{2}}{\dot{z}_{1}}\right)-u_{2} \sqrt{\dot{z}_{1}^{2}+\dot{z}_{2}^{2}} \sin \left(\arctan \frac{\dot{z}_{2}}{\dot{z}_{1}}\right) \\
& \ddot{z}_{2}=u_{1} \sin \left(\arctan \frac{\dot{z}_{2}}{\dot{z}_{1}}\right)+u_{2} \sqrt{\dot{z}_{1}^{2}+\dot{z}_{2}^{2}} \cos \left(\arctan \frac{\dot{z}_{2}}{\dot{z}_{1}}\right) .
\end{aligned}
$$

Since the expressions of new inputs $u_{1}$ and $u_{2}$ in (3) are derived from the system equations (2), the state transformation ensures that (3) satisfies the equations (4). By replacing $\ddot{z}_{1}$ and $\ddot{z}_{2}$ in (3) with $u_{x}$ and $u_{y}$, respectively:

$$
u_{1}=\frac{\dot{z}_{1} u_{x}+\dot{z}_{2} u_{y}}{\sqrt{\dot{z}_{1}^{2}+\dot{z}_{2}^{2}}}, u_{2}=\frac{\dot{z}_{1} u_{y}-u_{x} \dot{z}_{2}}{\dot{z}_{1}^{2}+\dot{z}_{2}^{2}}
$$

where $u_{x}$ and $u_{y}$ are parameters to be designed, and substituting (5) into the system equations (4), one gets

$$
\ddot{z}_{1}=u_{x}, \ddot{z}_{2}=u_{y} \text {. }
$$

Actually, if only the states of the original system (2), $[x, y, v, \theta]^{T}$, can be estimated, using the relationship of state transformation, the inputs (5) can be rewritten as

$$
u_{1}=u_{x} \cos \theta+u_{y} \sin \theta, u_{2}=\left(u_{y} \cos \theta-u_{x} \sin \theta\right) / v \text {. }
$$




\subsection{Dynamic model}

By ignoring the mass of the wheels, the equations of motion can be derived using Euler-Lagrange method as

$$
M(\mathbf{q}) \ddot{\mathbf{q}}+\bar{C}(\mathbf{q}, \dot{\mathbf{q}}) \dot{\mathbf{q}}=E(\mathbf{q}) \tau-C^{T}(\mathbf{q}) \lambda
$$

where

$$
\begin{gathered}
M=\left[\begin{array}{ccc}
m & 0 & -d m \sin \theta \\
0 & m & d m \cos \theta \\
-d m \sin \theta & d m \cos \theta & d^{2} m+I
\end{array}\right], \tau=\left[\begin{array}{l}
\tau_{r} \\
\tau_{l}
\end{array}\right] \\
\bar{C}=\left[\begin{array}{lll}
0 & 0 & -d m \dot{\theta} \cos \theta \\
0 & 0 & -d m \dot{\theta} \sin \theta \\
0 & 0 & 0
\end{array}\right], \quad E=\frac{1}{r}\left[\begin{array}{cc}
\cos \theta & \cos \theta \\
\sin \theta & \sin \theta \\
b & -b
\end{array}\right] .
\end{gathered}
$$

Here, $m$ is the robot mass, $I$ the moment of inertia of the robot about its center of mass, $d$ the distance between the center of mass and the center of the wheel axle, $r$ the wheel radius, $b$ half distance between the two wheels, $\tau_{l}$ and $\tau_{r}$ the motor torques on the wheels, and $\lambda$ the constraint force.

By differentiating (1), one gets $\ddot{\mathbf{q}}=\dot{S} \mathbf{v}+S \dot{\mathbf{v}}$. Then by substituting $\ddot{\mathbf{q}}$ into (8), pre-multiplying by $S^{T}$, and using the property $S^{T} C^{T}=0$, one can have

$$
\dot{\mathbf{v}}=-\left(S^{T} M S\right)^{-1} S^{T}(M \dot{S}+\bar{C} S) \mathbf{v}+\left(S^{T} M S\right)^{-1} S^{T} E \tau \text {. (10) }
$$

This can finally be calculated as $\dot{\mathbf{v}}=A(\mathbf{v})+B \boldsymbol{\tau}$, where

$$
A(\mathbf{v})=\left[\begin{array}{c}
d \omega^{2} \\
-\frac{d m \omega v}{d^{2} m+I}
\end{array}\right], B=\left[\begin{array}{cc}
\frac{1}{m r} & \frac{1}{m r} \\
\frac{b}{r\left(d^{2} m+I\right)} & -\frac{b}{r\left(d^{2} m+I\right)}
\end{array}\right] \text {. }
$$

After introducing an input transformation $\overline{\mathbf{u}}=\left[\bar{u}_{1}, \bar{u}_{2}\right]^{T}$ $=A(\mathbf{v})+B \boldsymbol{\tau}$, the dynamic model can be written as

$$
\dot{\mathbf{q}}=S(\mathbf{q}) \mathbf{v}, \quad \dot{\mathbf{v}}=\overline{\mathbf{u}}
$$

Here, we can also choose the center position of the wheel axle $(x, y)$ as the flat outputs $\left(z_{1}, z_{2}\right)$ and then all state variables and inputs can be expressed in terms of the flat outputs and their derivatives. The expression of state $\omega$ contains the second order derivatives of both the flat outputs, $\ddot{z}_{1}$ and $\ddot{z}_{2}$, which means that the system (11) need to be extended to 6 dimensions so that it can be transformed into a normal form via a state transformation. On applying one prolongation of $\bar{u}_{1}$, the extended system is given by

$$
\begin{array}{lll}
\dot{x}=v \cos \theta & \dot{v}=\bar{u}_{1} & \dot{\bar{u}}_{1}=\tilde{u}_{1} \\
\dot{y}=v \sin \theta & \dot{\theta}=\omega & \dot{\omega}=\tilde{u}_{2}
\end{array}
$$

where $\tilde{u}_{1}$ and $\tilde{u}_{2}=\bar{u}_{2}$ are new inputs of the extended system. The state transformation can be written as $\left[x, y, v, \theta, \bar{u}_{1}, \omega\right]^{T} \leftrightarrow\left[z_{1}, z_{2}, \dot{z}_{1}, \dot{z}_{2}, \ddot{z}_{1}, \ddot{z}_{2}\right]^{T}$. Now, we calculate the third order derivatives of the flat outputs directly using (12) as

$$
\left[\begin{array}{c}
\dddot{z}_{1} \\
\dddot{z}_{2}
\end{array}\right]=C+D\left[\begin{array}{l}
\tilde{u}_{1} \\
\tilde{u}_{2}
\end{array}\right]
$$

where

$$
C=\left[\begin{array}{c}
-2 \bar{u}_{1} \omega \sin \theta-v \omega^{2} \cos \theta \\
2 \bar{u}_{1} \omega \cos \theta-v \omega^{2} \sin \theta
\end{array}\right], D=\left[\begin{array}{cc}
\cos \theta & -v \sin \theta \\
\sin \theta & v \cos \theta
\end{array}\right] .
$$

By replacing $\dddot{z}_{1}$ and $\dddot{z}_{2}$ with $\bar{u}_{x}$ and $\bar{u}_{y}$, respectively, this yields

$$
\left[\begin{array}{l}
\tilde{u}_{1} \\
\tilde{u}_{2}
\end{array}\right]=D^{-1}\left(\left[\begin{array}{l}
\bar{u}_{x} \\
\bar{u}_{y}
\end{array}\right]-C\right)
$$

\section{Design of Control Law}

\subsection{Introduction of PD-spectral theory ${ }^{10}$}

Consider SISO LTV systems represented by the $n$ thorder scalar LTV dynamical systems of the form:

$$
y^{(n)}+\alpha_{n}(t) y^{(n-1)}+\cdots+\alpha_{2}(t) \dot{y}+\alpha_{1}(t) y=0 .
$$

It can be conveniently represented as $D_{\alpha}\{y\}=0$ using the scalar polynomial differential operator (SPDO)

$$
D_{\alpha}=\delta^{n}+\alpha_{n}(t) \delta^{n-1}+\cdots+\alpha_{2}(t) \delta+\alpha_{1}(t)
$$

where $\delta=d / d t$ is the derivative operator. The factorization of SPDO can be represented as

$$
D_{\alpha}=\left(\delta-\lambda_{n}(t)\right) \cdots\left(\delta-\lambda_{2}(t)\right)\left(\delta-\lambda_{1}(t)\right)
$$

where a collection $\left\{\lambda_{k}(t)\right\}_{k=1}^{n}$ is called a series Dspectrum(SD-spectrum) for $D_{\alpha}$ and an n-parameter family $\left\{\rho_{k}(t)=\lambda_{1, k}(t)\right\}_{k=1}^{n}$ is called a parallel Dspectrum(PD-spectrum) for $D_{\alpha}$, where $\lambda_{1, k}(t)$ are $n$ particular solutions for $\lambda_{1}(t)$ satisfying some nonlinear independent constrains. Actually, $\left\{y_{k}(t)=\right.$ $\left.\exp \left(\int \rho_{k}(t) \mathrm{d} t\right)\right\}_{k=1}^{n}$ constitutes a fundamental set of solutions to $D_{\alpha}\{y\}=0$.

The solution to $D_{\alpha}\{y\}=0$ is uniformly asymptotically stable if

(i) all PD-eigenvalues are of polynomial order or slower, that is, an integer $m>0$ exists such that $\lim _{t \rightarrow \infty} \frac{\rho_{k}(t)}{t^{m}}=0$; 
(ii) the extended means of real parts of PD-eigenvalues, that is $\operatorname{em}\left(\operatorname{Re} \rho_{k}(t)\right)=\lim _{T \rightarrow \infty} \frac{1}{T} \int_{t_{0}}^{t_{0}+T} \operatorname{Re} \rho_{k}(t) \mathrm{d} t$, are all negative.

\subsection{Control law of kinematic model}

If the desired trajectory of flat outputs $\left(z_{1}, z_{2}\right)$ are given by $\left(z_{1 d}(t), z_{2 d}(t)\right)$, tracking error can be introduced as $\mathbf{e}=\left[e_{1}, e_{11}, e_{2}, e_{21}\right]^{T}=\left[z_{1}-z_{1 d}, \dot{z}_{1}-\dot{z}_{1 d}, z_{2}-z_{2 d}, \dot{z}_{2}-\dot{z}_{2 d}\right]^{T}$. Using (6), parameters $u_{x}$ and $u_{y}$ can be designed as

$$
\begin{aligned}
& u_{x}=\ddot{z}_{1 d}-\alpha_{1 x}(t) e_{1}-\alpha_{2 x}(t) e_{11} \\
& u_{y}=\ddot{z}_{2 d}-\alpha_{1 y}(t) e_{2}-\alpha_{2 y}(t) e_{21}
\end{aligned}
$$

which yields

$$
\begin{aligned}
& \ddot{e}_{1}+\alpha_{2 x}(t) \dot{e}_{1}+\alpha_{1 x}(t) e_{1}=0 \\
& \ddot{e}_{2}+\alpha_{2 y}(t) \dot{e}_{2}+\alpha_{1 y}(t) e_{2}=0 .
\end{aligned}
$$

PD-spectral theory is used to design these time-varying control gains to ensure the error dynamics to be uniformly asymptotically stable.

First appropriate time-varying PD-eigenvalues which satisfy (i) and (ii) are designed as $\rho_{1}$ and $\rho_{2}$. Then corresponding SD-eigenvalues $\lambda_{1}$ and $\lambda_{2}$ can be calculated as $\lambda_{1}=\rho_{1}, \lambda_{2}=\rho_{2}+\frac{\dot{\rho}_{2}-\dot{\rho}_{1}}{\rho_{2}-\rho_{1}}$. Using (18), we obtain $\quad \alpha_{1 x}(t)=\lambda_{2} \lambda_{1}-\dot{\lambda}_{1}=\rho_{1} \rho_{2}+\frac{\rho_{1} \dot{\rho}_{2}-\rho_{2} \dot{\rho}_{1}}{\rho_{2}-\rho_{1}}$, $\alpha_{2 x}(t)=-\lambda_{2}-\lambda_{1}=-\rho_{1}-\rho_{2}-\frac{\dot{\rho}_{2}-\dot{\rho}_{1}}{\rho_{2}-\rho_{1}} \quad . \quad \alpha_{1 y}(t) \quad$ and $\alpha_{2 y}(t)$ can be designed in the same way.

\subsection{Control law of dynamic model}

Similarly, if the desired trajectory of flat outputs are given by $\left(z_{1 d}(t), z_{2 d}(t)\right)$ and tracking error $\mathbf{e}=\left[e_{1}, e_{11}, e_{12}, e_{2}, e_{21}, e_{22}\right]^{T}=\left[z_{1}-z_{1 d}, \dot{z}_{1}-\dot{z}_{1 d}, \ddot{z}_{1}-\ddot{z}_{1 d}, z_{2}\right.$ $\left.-z_{2 d}, \dot{z}_{2}-\dot{z}_{2 d}, \ddot{z}_{2}-\ddot{z}_{2 d}\right]^{T}$, then parameters $\bar{u}_{x}$ and $\bar{u}_{y}$ can be designed as

$$
\begin{aligned}
& \bar{u}_{x}=\dddot{z}_{1 d}-\alpha_{1 x}(t) e_{1}-\alpha_{2 x}(t) e_{11}-\alpha_{3 x}(t) e_{12} \\
& \bar{u}_{y}=\dddot{z}_{2 d}-\alpha_{1 y}(t) e_{2}-\alpha_{2 y}(t) e_{21}-\alpha_{3 y}(t) e_{22} .
\end{aligned}
$$

By designing appropriate PD-eigenvalues $\rho_{1}, \rho_{2}$ and $\rho_{3}$, SD-eigenvalues can be calculated as $\lambda_{1}=\rho_{1}$, $\lambda_{2}=\rho_{2}+\frac{\dot{V}_{2}}{V_{2}}, \lambda_{3}=\rho_{3}+\frac{\dot{V}_{3}}{V_{3}}-\frac{\dot{V}_{2}}{V_{2}}$ where $V_{2}=\rho_{2}-\rho_{1}$,
$V_{3}=\operatorname{det}\left[\begin{array}{ccc}1 & 1 & 1 \\ \rho_{1} & \rho_{2} & \rho_{3} \\ \rho_{1}^{2}+\dot{\rho}_{1} & \rho_{2}^{2}+\dot{\rho}_{2} & \rho_{3}^{2}+\dot{\rho}_{3}\end{array}\right]$. and then control gains are obtained by $\alpha_{1 x}(t)=-\lambda_{3} \lambda_{2} \lambda_{1}+\lambda_{3} \dot{\lambda}_{1}+\dot{\lambda}_{2} \lambda_{1}+$ $\lambda_{2} \dot{\lambda}_{1}-\ddot{\lambda}_{1}, \alpha_{2 x}(t)=\lambda_{3} \lambda_{2}+\lambda_{3} \lambda_{1}+\lambda_{2} \lambda_{1}-\dot{\lambda}_{2}-2 \dot{\lambda}_{1}, \alpha_{3 x}(t)$ $=-\lambda_{3}-\lambda_{2}-\lambda_{1} \cdot \alpha_{1 y}(t), \alpha_{2 y}(t)$ and $\alpha_{3 y}(t)$ can be designed in the same way.

\section{Simulation Result}

The desired trajectory is given by $z_{1 d}(t)=3.75 \times$ $10^{-4} t^{3}-1.125 \times 10^{-2} t^{2}+0.15 t(\mathrm{~m}), z_{2 d}(t)=-3 \times 10^{-4} t^{3}+$ $9 \times 10^{-3} t^{2}(\mathrm{~m})$, over $t=[0,20](\mathrm{s})$.

In the kinematic model, initial states are set as $[x(0), y(0), v(0), \theta(0)]=[-0.1,0.1,0.05,-0.5]$. It should be pointed out that the additional state $v$ is in the controller. PD-eigenvalues are selected as $\rho_{1}=-(1+$ $0.25 t)+(1+0.25 t) i, \rho_{2}=-(1+0.25 t)-(1+0.25 t) i$. Fig. 2(a) shows that the mobile robot gradually converges to
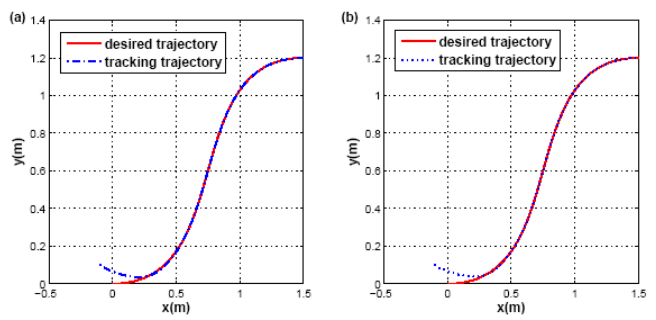

Fig.2. Desired trajectory and tracking trajectory: (a)Kinematic model. (b)Dynamic model.
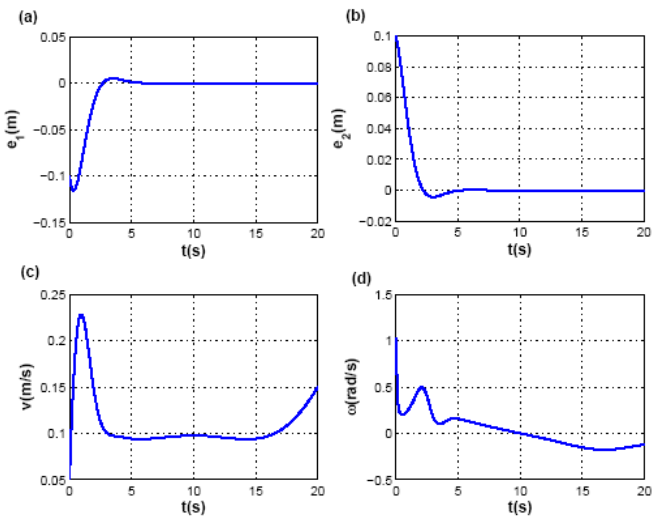

Fig.3. Kinematic model: (a)Tracking error $e_{1}$. (b)Tracking error $e_{2}$. (c)Heading speed $v$. (d)Turning speed $\omega$. 

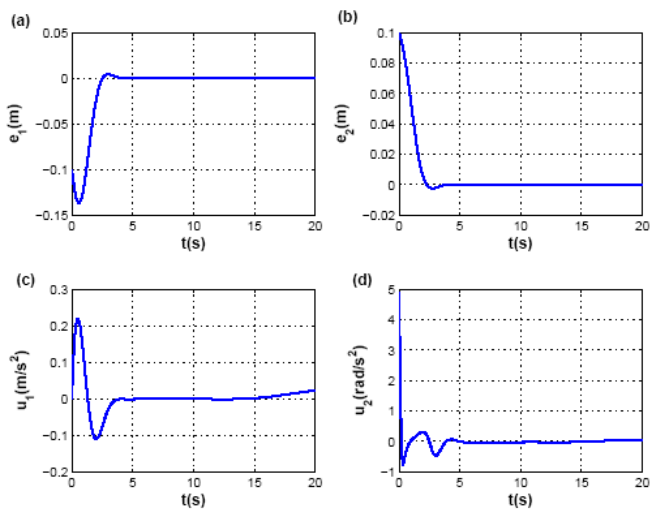

Fig.4. Dynamic model: (a)Tracking error $e_{1}$. (b)Tracking error $e_{2}$. (c)Transformed input $\bar{u}_{1}$. (d)Transformed input $\bar{u}_{2}$.

the desired trajectory and finally moves along it. Fig. 3 demonstrates the tracking errors $e_{1}, e_{2}$ which converge to zero and the control inputs $v$ and $\omega$.

In the dynamic model, $\left[x(0), y(0), v(0), \theta(0), \bar{u}_{1}(0)\right.$, $\omega(0)]=[-0.1,0.1,0.05,-0.5,0,0]$. Controller is designed with PD-eigenvalues $\rho_{1}=-(1+0.25 t)+(1+0.25 t) i$, $\rho_{2}=-(1+0.25 t)-(1+0.25 t) i, \rho_{3}=-2-0.25 t$. Fig. 2(b) shows the tracking trajectory and Fig. 4 depicts the tracking errors and the transformed inputs $\bar{u}_{1}$ and $\bar{u}_{2}$.

\section{Conclusion}

In this paper, we have presented a novel method for trajectory tracking control of a two-wheeled mobile robot in its kinematic model and dynamic model. Based on differential flatness, the systems can be transformed into normal forms to use feedback linearization. The application of PD-spectral theory ensures the stability of the tracking error dynamics and establishes adjustable poles to change as we want.

\section{Acknowledgements}

This work was supported by the National Basic Research Program of China (973 Program: 2012CB821200, 2012CB821201) and the NSFC (61134005, 61221061, 61327807).

\section{References}

1. R. Fierro and F. V. Lewis, Control of a non-holonomic mobile robot: backstepping kinematics into dynamics, $J$. Robot. Syst., 14(3) (1997) 149-163.

2. J. M. Yang and J. H. Kim, Sliding mode control for trajectory tracking of nonholonomic wheeled mobile robots, IEEE Trans. Robot. Autom., 15(3) (1999) 578-587.

3. D. H. Kim and J. H. Oh, Tracking control of a twowheeled mobile robot using input-output linearization, Contr. Eng. Pract., 7(3) (1999) 369-373.

4. V. Boquete, R. Garcia, R. Barea, and M. Mazo, Neural control of the movements of a wheel-chair, J. Intell. Robot. Syst., 25(3) (1999) 213-226.

5. T. Das and I. N. Kar, Design and implementation of an adaptive fuzzy logic-based controller for wheeled mobile robots, IEEE Trans. Contr. Syst. Technol., 14(3) (2006) 501-510.

6. Chun-Hsu Ko and Sunil K. Agrawal, Walk-assist robot: a novel approach to gain selection of a braking controller using differential flatness, American Control Conference (Marriott Waterfront, Baltimore, 2010), pp. 2799-2804.

7. Chin Pei Tang, Differential flatness-based kinematic and dynamic control of a differentially driven wheeled mobile robot, in Proc. IEEE Int. Conf. Robotics and Biomimetics (Guilin, China, 2009), pp. 2267-2272.

8. Ji-Chul Ryu and Sunil K. Agrawal, Differential flatnessbased robust control of a two-wheeled mobile robot in the presence of slip, in Proc. DSCC ASME Dynamic Systems and Control Conference (Ann Arbor, Michigan, USA, 2008), pp. 1-7.

9. M. Fliess, J. Lévine, Ph. Martin, and P. Rouchon, Flatness and defect of non-linear systems: introduction theory and examples, International Journal of Control, 61(6) (1995) 1327-1361.

10. J. Zhu, A unified spectral theory for linear time-varying systems--progress and challenges, in Proc. IEEE Conf. Decision and Control (New Orleans, LA, 1995), pp. 25402546. 